\title{
IS-2: The Effect of External Perturbations on Ecological Oscillators
}

\author{
Eli E. Goldwyn ${ }^{1, *}$ \\ ${ }^{1}$ Department of Mathematics, University of Portland, Portland, OR 97203 \\ goldwyn@up.edu
}

Population interactions cause oscillations across a variety of ecological systems. These ecological population oscillators are subject to external forces including climatic effects and migration. One way of understanding the impact of these perturbations is to study their effect on the phase of the oscillations. In this talk, we introduce tools commonly used to describe the phase of oscillating phenomena in cardiac dynamics, circadian rhythms, firing neurons, and more recently, coupled predator-prey dynamics. We discuss applications of these methods to several common ecological models and present undergraduate research opportunities to extend these techniques to other models to address specific ecological issues and phenomena 